\title{
EXTENSIONS OF CERTAIN COMPACT OPERATORS ON VECTOR-VALUED CONTINUOUS FUNCTIONS
}

\author{
SURJIT SINGH KHURANA
}

(Communicated by John B. Conway)

\begin{abstract}
For any compact Hausdorff spaces $X, Y$ with $\varphi: X \rightarrow Y$ a continuous onto mapping, $E, F$, Hausdorff locally convex spaces with $F$ complete, $C(X, E)(C(Y, E))$ all $E$-valued continuous functions on $X(Y)$, and $L: C(Y, E) \rightarrow F$ a $\tau$-compact continuous operator $\left(\sigma\left(F, F^{\prime}\right) \leq \tau \leq \tau\left(F, F^{\prime}\right)\right)$, it is proved there exists a $\tau$-compact continuous operator $L_{0}: C(X, E) \rightarrow F$ such that $L_{0}(f \circ \varphi)=L(f)$ for every $f \in C(Y, E)$.
\end{abstract}

In this paper $X, Y$ are compact Hausdorff spaces, $\varphi: X \rightarrow Y$ a continuous onto function, $E, F$ Hausdorff locally convex spaces over $K$, the field of real or complex numbers, and $C(X, E)$ (resp. $C(Y, E)$ ) all continuous $E$-valued functions on $X$ (resp. $Y$ ). The space $C(Y, E) \circ \varphi$ is a subspace of $C(X, E)$. When $E, F$ are Banach spaces it is proved in $[\mathbf{1}, \mathbf{2}]$ that every weakly compact operator $T: C(Y, E) \rightarrow$ $F$ has extension to a weakly compact operator $T_{0}: C(X, E) \rightarrow F$ in the sense that $T_{0}(f \circ \varphi)=T(f)$ for every $f \in C(Y, E)$. Here we will prove the result for general locally convex spaces $E, F$ assuming $F$ to be complete, by using the measure extension techniques discussed in [8]. On $C(X, E)$ or $C(Y, E), u$ will denote the uniform topology. For locally convex spaces $G_{1}, G_{2}$, an operator $T: G_{1} \rightarrow G_{2}$ will be called compact if bounded sets of $G_{1}$ are mapped into relatively compact subsets of $G_{2}$. For locally convex spaces we refer to [9]. $\mathcal{L}(E, F)$ will denote the space of all continuous linear operators from $E$ to $F$. Let $\left\{|\cdot|_{p}: p \in P\right\}$ be the family of all continuous seminars on $E$. $M(X), M(Y)$ will denote all regular scalar Borel measures on $X$ and $Y$ resp. If $T:(C(Y, E), u) \rightarrow F$ is continuous and $f \in F^{\prime}$, then $f \circ T \in(C(Y, E), u)^{\prime}$ and so $[\mathbf{5}, \mathbf{7}]$ there exists $p \in P$ such that $|f \circ T|_{p} \in M^{+}(Y)$ (note for $g \in C(Y), g \geq 0,|(f \circ T)|_{p}(g)=\sup \{|(f \circ T)(h)|: h \in C(Y, E)$ and $\left.\|h\|_{p} \leq f\right\}$, where $\left.\|h\|_{p}(y)=\|h(y)\|_{p}[7]\right)$. Here $C(Y)$ stands for all $K$-valued continuous functions on $Y$. Also $(C(Y, E), u)^{\prime}=M\left(Y, E^{\prime}\right)[\mathbf{5}, \mathbf{7}] . B(X), B(Y)$ will denote all Borel subsets of $X$ and $Y$ respectively. For an algebra $\mathfrak{A}$ of subsets of a set $Z, S(\mathfrak{A})$ will denote all $K$-valued $\mathfrak{A}$-simple functions on $Z$.

THEOREM. Assume $F$ is a complete locally convex space, and let $\tau$ be another locally convex topology on $F$ such that $\sigma\left(F, F^{\prime}\right) \leq \tau \leq \tau\left(F, F^{\prime}\right)$. Let $L:(C(Y, E), u)$ $\rightarrow F$ be a continuous $T$-compact operator, i.e., bounded subsets of $C(Y, E)$ are mapped into relatively $\tau$-compact subsets of $F$. Then there exists a continuous

Received by the editors July 1, 1986 and, in revised form, October 16, 1986.

1980 Mathematics Subject Classification (1985 Revision). Primary 28B05, 46G10, 47B05; Secondary 46A22, 47B38.

Key words and phrases. Submeasures, exhaustive submeasures, regular group-valued Borel measures, compact operators. 
$\tau$-compact operator $L_{0}:(C(X, E), u) \rightarrow F$ such that $L_{0}(f \circ \varphi)=L(f)$ for each $f \in C(Y, E)$.

ProOF. $\mathcal{L}(E, F)$ is the space of all continuous linear operators from $E$ to $F$. Let $\tilde{F}$ be the weak completion of $F$ and $G$ the space of all continuous linear operators from $E$ into $\left(\tilde{F}, \sigma\left(\tilde{F}, F^{\prime}\right)\right)$. For any finite subset $H \subset F^{\prime}$ and any bounded $B \subset E$, a seminorm $m$ is generated on $G$ :

$$
m(Q)=\sup \{|f \circ Q(x)|: x \in B, f \in H\},
$$

$Q \in G$. Under the locally convex topology generated by these seminorms, $G$ is a complete locally convex space. The topology on $\mathcal{L}(E, F)$ is the one induced by $G$. Since $L$ is weakly compact, we get $[\mathbf{3}, \mathbf{5}]$ a regular Borel measure $\mu_{1}: B(Y) \rightarrow$ $\mathcal{L}(E, F)$ with the properties:

(I) For any $f \in F^{\prime}, f \circ \mu_{1}=f \circ L$.

(II) For any equicontinuous set $H \subset F^{\prime}$, there exists a $p \in P$ such that

$$
\sup \left\{\left|\sum f\left(\mu_{1}\left(A_{i}\right)\left(x_{i}\right)\right)\right|\right\}<\infty,
$$

where the supremum is taken over $f \in H$, all finite Borel partitions $\left\{A_{i}\right\}$ of $Y$, and all $x_{i} \in E$ satisfying $\left|x_{i}\right|_{p} \leq 1$.

(III) For any bounded set $B \subset E,\left\{\sum \mu_{1}\left(A_{i}\right) x_{i}\right\}$, where $\left\{A_{i}\right\}$ varies over all finite disjoint collections of Borel subsets of $X$ and $x_{i} \in B$, is relatively $\tau$-compact in $F$.

Let $\left\{|\cdot|_{s}: s \in S\right\}$ be the family of all continuous seminorms on $G$. In the notation of $\left[8\right.$, p. 160], let $\mathcal{U}=\left\{\varphi^{-1}(A): A \in B(Y)\right\}$; defines $\mu: \mathcal{U} \rightarrow \mathcal{L}(E, F), \mu\left(\varphi^{-1}(A)\right)=$ $\mu_{1}(A), A \in B(Y)$. Each $s \in S$ gives an exhaustive, order $\sigma$-continuous submeasure $\dot{\mu}_{s}: U \rightarrow[0, \infty), \dot{\mu}_{s}(B)=\sup \left\{|\mu(A)|_{s}: A \in \mathcal{U}, A \subset B\right\}$ for every $B \in \mathcal{U}$. As in the proof of $[8$, Theorem 1, pp. 160-162], these submeasures can be extended to exhaustive, order $\sigma$-continuous, regular submeasures $\bar{\mu}_{s}: B(X) \rightarrow[0, \infty)$ with the properties

(i) for any $s(1), s(2) \in S, \dot{\mu}_{s(1)} \leq \dot{\mu}_{s(2)}$ implies $\bar{\mu}_{s(1)} \leq \bar{\mu}_{s(2)}$,

(ii) for $\varepsilon>0, s \in S$ and $B \in B(X)$, there exists $B_{0} \in \mathcal{U}$, such that $\bar{\mu}_{s}\left(B \triangle B_{0}\right)<$ $\varepsilon$ (here $\left.B \triangle B_{0}=\left(B \backslash B_{0}\right) \cup\left(B_{0} \backslash B\right)\right)$.

On $B(X)$ we define $F-N$ topology $\mathcal{F}$ generated by $\left\{\bar{\mu}_{s}: s \in S\right\}[4$, p. 271]. $B(X)$ becomes a topological ring in which $U$ is dense. This means the uniformly continuous mapping $\mu: \mathcal{U} \rightarrow G$ can be uniquely extended to a uniformly continuous mapping $\mu_{0}: B(X) \rightarrow G$. This $\mu$ is countably additive and regular [8].

We shall prove some properties of $\mu_{0}$.

(a) First we prove that (II) holds when $\left\{A_{i}\right\}$ are chosen from $B(X)$. Take any equicontinuous $H \subset F^{\prime}$. By (II) above there exists a $p \in P$ and $M, 0<M<$ $\infty$, such that $\sup \left\{\left|\sum f\left(\mu\left(A_{i}\right)\left(x_{i}\right)\right)\right|:\left\{x_{i}\right\}\right.$ a finite subset of $E$ with $p\left(x_{i}\right) \leq 1$, and $\left\{A_{i}\right\}$ a disjoint collection in $\left.U\right\} \leq M$, for each $f \in H$. Fix a finite subset $\left\{x_{i}: 1 \leq i \leq n\right\}$ in $E$ with $p\left(x_{i}\right) \leq 1$ for each $i$, and a finite disjoint collection $\left\{B_{i}\right\}$ in $B(X)$. Take nets in $\mathcal{U}, A_{\alpha}^{i} \rightarrow B_{i}$ in $(B(X), \mathcal{F})$. Put $C_{\alpha}^{1}=A_{\alpha}^{1}, C_{\alpha}^{i}=$ $A_{\alpha}^{i} \backslash \bigcup_{j=1}^{i-1} A_{\alpha}^{j}, i \geq 2$. Then $\left\{C_{\alpha}^{i}: 1 \leq i \leq n\right\}$ are mutually disjoint and $C_{\alpha}^{i} \rightarrow B_{i}$. From $\sup \left\{\left|\sum f\left(\mu\left(C_{\alpha}^{i}\right)\left(x_{i}\right)\right)\right|: f \in H,\left\{x_{i}\right\} \subset E\right.$ with $\left.p\left(x_{i}\right) \leq 1\right\} \leq M$, we get

$$
\sup \left\{\sum\left|f\left(\mu_{0}\left(B_{i}\right)\left(x_{i}\right)\right)\right|: f \in H,\left\{x_{i}\right\} \subset E\right\} \leq M
$$

for $p\left(x_{i}\right) \leq 1$ and $\left\{B_{i}\right\}$ a disjoint finite collection in $B(X)$. 
Now we claim that $\mu_{0}(B) \in \mathcal{L}(E, F)$ for every $B \in B(X)$. Take a net $\left\{A_{\alpha}\right\}$ in $\mathcal{U}$ such that $A_{\alpha} \rightarrow B$ in $(B(X), \mathcal{F})$. Fix $x \in E$. Since $\left\{\mu\left(A_{\alpha}\right)(x)\right\}_{\alpha}$ is a relatively $\tau$-compact set we get $\mu_{0}(A)(x) \in F$. Using what is proved first in (a) we get $\mu_{0}(B) \in \mathcal{L}(E, F)$.

(b) Proceeding as in (a) we prove that (III) holds when $\left\{A_{i}\right\}$ are chosen from $B(X)$.

Because of properties (a) and (iv), the mapping $\mu_{0}: B(X) \rightarrow \mathcal{L}(E, F)$ gives a $\tau$-compact linear continuous mapping $L_{1}:(S(B(X)) \otimes E, u) \rightarrow F$. The completion of $(S(B(X)) \otimes E, u)$ contains $C(X) \otimes E$ and therefore also contains $C(X, E)$ (note $C(X) \otimes E$ is dense in $(C(X, E), u))$. Thus we get a continuous $\tau$-compact operator $L_{0}: C(X, E) \rightarrow F$ (note $F$ is complete). For an $f \in C(Y), x \in E$, and $g \in F^{\prime}$, $g \circ L(f \otimes x)=g \circ \mu_{1}(f \otimes x)$. Take a sequence $\left\{f_{n}\right\}$ in $S(B(Y))$, such that $f_{n} \rightarrow f$ uniformly on $Y$. Thus $\left\{f_{n} \circ \varphi\right\} \subset S(\mathcal{U})$ and $f_{n} \circ \varphi \rightarrow f \circ \varphi$ uniformly on $X$. This means

$$
\begin{aligned}
g \circ L_{0}(f \circ \varphi \otimes x) & =\lim g \circ L_{1}\left(f_{n} \circ \varphi \otimes x\right)=\lim g \circ \mu_{1}\left(f_{n} \otimes x\right) \\
& =g \circ \mu_{1}(f \otimes x)=g \circ L(f \otimes x) .
\end{aligned}
$$

Thus $L_{0}(f \circ \varphi)=L(f)$. Since $C(Y) \otimes E$ is dense in $(C(Y, E), u)$ we get $L_{0}(f \circ \varphi)=$ $L(f)$ for every $f \in C(Y, E)$. This proves the theorem.

I am thankful to the referee for some useful suggestions.

\section{REFERENCES}

1. F. Bombal, On weakly compact operators on spaces of vector-valued continuous functions, Proc. Amer. Math. Soc. 97 (1986), 93-96.

2. F. Bombal and B. Rodriguez-Salinas, Some classes of operators on $C(K, E)$. Extensions and applications, Arch. Math. 47 (1986), 55-65.

3. J. K. Brooks and P. W. Lewis, Linear operators and vector measures, Trans. Amer. Math. Soc. 192 (1974), 139-162.

4. L. Drewnowski, Topological rings of sets, continuous set functions, integration. I, II, Bull. Acad. Polon. Sci. Sér. Sci. Math. Astronom. Phys. 20 (1972), 269-286.

5. A. Katsaras and D. Lin, Integral representations of weakly compact operators, Pacific J. Math. 56 (1975), 547-556.

6. S. S. Khurana, Topologies on spaces of vector-valued continuous functions, Trans. Amer. Math. Soc. 241 (1978), 195-211.

7. $\ldots$, Topologies on spaces of vector-valued continuous functions. II, Math. Ann. 234 (1978), 159-166.

8. __ Extensions of group-valued regular Borel measure, Math. Nachr. 97 (1980), 159-165.

9. H. H. Schaeffer, Topological vector spaces, Macmillan, New York, 1966.

DePARTMENT OF MAThematics, UNiVersity of IOWA, IOWA City, IOWA 52242 